Rev. Adm. Saúde (On-line), São Paulo, v. 20, n. 81: e261, out. - dez. 2020, Epub 24 dez. 2020

http://dx.doi.org/10.23973/ras.81.261

ARTIGO DE REVISÃO

\title{
Assistência à saúde em pediatria: uma revisão integrativa sobre os cuidados paliativos
}

Health care in pediatrics: an integrative review on palliative care

\author{
Luciana Alves Silveira Monteiro1, Camila Correia Oliveira², Marília Aguiar³, \\ Claudirene Milagres Araújo4, Raimundo Monteiro Correio ${ }^{5}$
}

1. Enfermeira, mestre em enfermagem. Enfermeira na UTI do Hospital das Clínicas da Universidade Federal de Minas Gerais, Belo Horizonte MG.

2. Enfermeira, especialista em enfermagem em UTI adulto e cuidados paliativos. Enfermeira na Unidade de Cuidados Paliativos do Hospital João Paulo II. Belo Horizonte MG.

3. Docente do curso de pós-graduação da Faculdade Ciências Médicas de Minas Gerais (FCM-MG). Belo Horizonte MG.

4. Enfermeira, mestre em enfermagem. Docente nos cursos de enfermagem e medicina da FCM-MG, Belo Horizonte MG.

5. Enfermeiro, especialista em enfermagem em unidade de Terapia intensiva adulto.

\section{RESUMO}

Introdução: cuidado paliativo é uma abordagem que promove qualidade de vida tanto dos pacientes que enfrentam doenças que ameacem a continuidade da vida, quando de seus familiares, por meio de aspectos da prevenção e alívio do sofrimento, requerendo a identificação precoce, avaliação e tratamento da dor. Pode ser realizado em diversos cenários, inclusive em pediatria. Objetivo: analisar artigos científicos disseminados em periódicos on-line acerca da temática cuidados paliativos em pediatria. Método: revisão integrativa da literatura, com coleta de dados no mês de agosto de 2020, nas bases de dados LILACS, SCIELO, MEDLINE/PubMed, IBECS. Resultados: foram selecionadas 14 publicações, cujas análises textuais permitiram a construção de quatro abordagens temáticas: 0 significado do treinamento no campo dos cuidados paliativos; cuidados paliativos e luto; a espiritualidade e o paciente sob cuidados paliativos e cuidados paliativos e aspectos éticos. Conclusão: o estudo verificou a relevância das dimensões do 
cuidado paliativo em pediatria durante a assistência e a necessidade do desenvolvimento de novos estudos para disseminar conhecimento sobre o tema.

Palavras-chave: Cuidados paliativos; Cuidados paliativos na terminalidade da vida; Atenção à saúde; Pediatria.

\begin{abstract}
Introduction: Palliative Care is an approach that promotes quality of life for both patients facing illnesses that threaten the continuity of life, and their family members, through aspects of prevention and relief of suffering, requiring early identification, evaluation, and treatment of ache. It can be performed in several settings, including pediatrics. Objective: to analyze scientific articles disseminated in online journals about the theme of palliative care in pediatrics. Method: integrative literature review, with data collection in August 2020, in the LILACS, SCIELO, MEDLINE / PubMed, IBECS databases. Results: 14 publications were selected; whose textual analysis allowed the construction of four thematic approaches: the meaning of training in the field of palliative care; palliative care and mourning; spirituality and the patient under palliative care and palliative care and ethical aspects. Conclusion: a study verified the relevance of the dimensions of palliative care in pediatrics during care and the need to develop new studies to disseminate knowledge on the topic.
\end{abstract}

Keywords: Palliative Care; Hospice Care; Health Care; Pediatrics.

\title{
INTRODUÇÃO
}

O cuidado em saúde implica em ações integrais que não se reduzem apenas aos aspectos técnicos assistenciais, permeia pela construção de vínculos, por meio da escuta dos sujeitos e o respeito pelo seu sofrimento e história de vida que compõem elementos inerentes à sua constituição. Sabe-se que, historicamente, o cuidado exige dos seres humanos uma atitude de solicitude, diligência, atenção, bom-trato, preocupação, inquietação e responsabilidade, requisitando uma busca incessante por atenção efetivamente direcionada a si mesmo ou ao outro. ${ }^{1}$

O cuidado perpassa por diversas esferas, sendo uma delas o paliativo, que, segundo a Organização Mundial de Saúde (OMS), é uma abordagem que promove a qualidade de vida tanto dos pacientes que enfrentam doenças que ameacem a continuidade da vida, quando de seus familiares, por meio de aspectos da prevenção e alívio do sofrimento, requerendo a identificação precoce, avaliação e tratamento de estímulos álgicos. ${ }^{2}$ 
Ademais, o cuidado paliativo é utilizado para designar uma forma de assistência interdisciplinar direcionada às pessoas que vivenciam doenças ameaçadoras à vida. Nessa perspectiva, o cuidado paliativo preconiza a preservação da autonomia do paciente, especialmente no tratamento de sintomas de caráter físico, social, psicológico e espiritual. ${ }^{2}$

O cuidado paliativo, quando utilizado precocemente, não apenas melhora a qualidade de vida dos pacientes, mas também é capaz de promover dignidade no cuidado, além de reduzir as hospitalizações desnecessárias e o uso de serviços de saúde, de modo a contribuir para a utilização eficiente dos recursos. ${ }^{3}$ Sabe-se que as estratégias dos cuidados paliativos ainda estão em processo de construção, motivo pelo qual a maior parte das ações ainda são desafiadoras, por isso, essa abordagem não se restringe à execução de procedimentos em pacientes, mas à propagação da preocupação, interesse, interação e compromisso pelo cuidado. ${ }^{4}$

Ressalta-se que as práticas de cuidados paliativos podem ser realizadas em diversos cenários, como em instituições hospitalares e de longa permanência para idosos; ambientes de terapia intensiva; ambulatórios especializados e no próprio domicílio. ${ }^{2,5,6}$

Nesse sentido, tem-se que a pediatria demanda ações paliativas intensas e contínuas em razão da necessidade premente de controle da dor e de outros sintomas que acometem crianças e adolescentes com desdobramentos na dinâmica familiar, uma vez que implica em uma reorganização da família que necessita conciliar a vida doméstica com as frequentes idas ao hospital ${ }^{7}$, além de frequentes e repetidas internações que afastam a criança e/ou o adolescente das atividades habituais, como escola e lazer. As mudanças na vida da criança, do adolescente e sua família, ao se depararem com a doença crônica, não englobam simplesmente alterações orgânicas ou físicas, mas atravessam esse angulo e promovem alterações emocionais e sociais em toda a família, as quais exigem constantes cuidados e adaptações. ${ }^{7}$

Dito isso, o objetivo desta pesquisa foi analisar artigos científicos disseminados em periódicos on-line acerca da temática cuidados paliativos em pediatria. Desse modo, entende-se ser o estudo desta temática de grande relevância para o campo da saúde, já que poderá estimular a equipe multiprofissional a refletir sobre a necessidade de atender ao público em pediatria e suas famílias que se encontram diante da ausência de tratamento curativo capaz de recuperar integralmente a saúde, mas que - independentemente de cura - requerem assistência que propicie alívio para o seu sofrimento físico e espiritual. Também poderá subsidiar novas investigações acerca da temática, visto que esta dimensão necessita ser mais explorada no âmbito acadêmico.

\section{MÉTODO}

Sabe-se o estudo de revisão integrativa é um método de pesquisa destacado como uma ferramenta de grande importância na área da saúde, pois proporciona busca, avaliação crítica e síntese de evidências sobre um tema investigado. ${ }^{8}$ Esses aspectos potencializam a identificação de resultados relevantes, bem como de 
lacunas capazes de direcionar rumo ao desenvolvimento de futuras pesquisas, além de auxiliar os profissionais na escolha de condutas e na tomada de decisões, desencadeando pensamento e saber crítico. ${ }^{8}$

Para organizar esta revisão, foi trilhado o percurso metodológico subdividido em seis fases: elaboração da questão norteadora; organização dos critérios de inclusão e exclusão e da busca na literatura; definição das informações a serem extraídas dos estudos selecionados; avaliação dos estudos incluídos; interpretação dos resultados e - finalmente - apresentação da revisão. Para conduzir este estudo, definiu-se a seguinte questão norteadora: Qual a produção científica existente acerca da temática cuidados paliativos e pediatria?

O levantamento bibliográfico foi realizado por meio de busca eletrônica nas seguintes bases de dados disponíveis na Biblioteca Virtual em Saúde: Literatura Latino-Americana e do Caribe em Ciências da Saúde (LILACS), USA National Library of Medicine (MEDLINE/PubMed) e Indice Bibliográfico Español de Ciencias de la Salud (IBECS) e na biblioteca eletrônica Scientific Eletronic Library Online (SciELO).

Foram incluídos artigos completos disponíveis eletronicamente, nos idiomas português, inglês e espanhol, no período de janeiro de 2015 a julho de 2020, e que apresentassem a temática proposta no título, no resumo ou nos descritores. Quando aos critérios de exclusão, foram excluídos: cartas ao editor, relatos de casos, editoriais, artigos em duplicidade, publicados em outros idiomas, com exceção do português, do inglês e do espanhol, que antecedessem o ano de 2015 e aqueles que não abordavam diretamente a temática proposta.

O levantamento dos artigos foi realizado no mês de agosto de 2020; como estratégias de investigação, foram utilizados os Descritores em Ciências da Saúde (DeCS): Cuidados Paliativos; Cuidados Paliativos na Terminalidade da Vida; Atenção à Saúde; Pediatria e suas variantes na língua inglesa e espanhola.

Após a seleção dos artigos, foram definidas as informações que seriam extraídas dos estudos. Para viabilizar a apreensão das informações, utilizou-se banco de dados elaborado no software Microsoft Office Excel 2013, composto das seguintes variáveis: título do artigo, ano de publicação, país, base de dados, título do periódico, delineamento do estudo, intervenção e desfecho. Os dados obtidos foram agrupados em quadros e em abordagens temáticas e interpretados com base na literatura.

\section{RESULTADOS}

O universo do estudo foi constituído por 69 publicações pertinentes à temática investigada, das quais 14 compuseram a amostra por atenderem aos critérios de inclusão. A maioria das publicações $(26,7 \%)$ data do ano de 2015, seguido por 2016 (20\%) e $2018(20 \%)$. Os países que mais produziram artigos sobre essa temática foram Brasil (46,7\%), Colômbia (20\%) e Estados Unidos (20\%). A maior parte dos estudos era quantitativo (40\%), após vem os estudos de revisão $(26,7 \%)$, 
atualização $(13,4 \%)$ e qualitativo $(13,4 \%)$, concentrando-se na base de dados SciELO (60\%) e MEDLINE/PubMed (20\%).

As produções acerca do tema abordado foram extraídas de periódicos que tratam de questões relacionadas aos cuidados de fim de vida, fator que explica o quantitativo de publicações encontradas no referido periódico. A distribuição completa dos artigos selecionados para a presente revisão está disposta no Quadro 1. 
Quadro 1. Distribuição dos estudos incluídos na revisão integrativa de acordo com título, ano, país, delineamento, intervenção e desfecho, dispostos em ordem decrescente de publicação.

\begin{tabular}{|c|c|c|c|c|c|}
\hline Título & Ano/País & $\begin{array}{l}\text { Base de } \\
\text { dados/Periódico }\end{array}$ & Estilo/Delineamento & Intervenção & Desfecho \\
\hline $\begin{array}{l}\text { Opinión de los } \\
\text { profesionales de la } \\
\text { salud sobre un } \\
\text { documento de registro } \\
\text { de directivas de } \\
\text { adecuación de } \\
\text { esfuerzo terapéutico } \\
\text { en pediatría }\end{array}$ & 2020/Uruguai & $\begin{array}{l}\text { SciELO/Revista } \\
\text { Médica del } \\
\text { Uruguay }\end{array}$ & $\begin{array}{l}\text { Quantitativo /Estudo } \\
\text { transversal com } \\
\text { entrevistas a médicos e } \\
\text { enfermeiras ( } n=248 \\
\text { entrevistados) }\end{array}$ & $\begin{array}{l}\text { Aplicação de questionário } \\
\text { estruturado, elaborado } \\
\text { especificamente para este } \\
\text { trabalho. }\end{array}$ & $\begin{array}{l}\text { A maioria dos profissionais } \\
\text { entrevistados havia participado no } \\
\text { cuidado de crianças com } \\
\text { necessidade de cuidados paliativos, } \\
\text { porém expressaram ter dúvidas } \\
\text { clínicas, éticas e/ou legais no que } \\
\text { tange a paliação. }\end{array}$ \\
\hline $\begin{array}{l}\text { Physicians' } \\
\text { knowledge about } \\
\text { patients' religious } \\
\text { beliefs in pediatric } \\
\text { care }\end{array}$ & 2019/Brasil & $\begin{array}{l}\text { SciELO/Revista } \\
\text { Paulista de } \\
\text { Pediatria }\end{array}$ & $\begin{array}{l}\text { Quantitativo /Estudo } \\
\text { transversal com } \\
\text { residentes em pediatria e } \\
\text { médicos pediatras ( } \mathrm{n}= \\
116 \text { entrevistados) }\end{array}$ & $\begin{array}{l}\text { Aplicação de questionário } \\
\text { estruturado, elaborado } \\
\text { especificamente para este } \\
\text { trabalho. }\end{array}$ & $\begin{array}{l}\text { Apesar de a maioria dos } \\
\text { profissionais dizer ser apta a lidar } \\
\text { com as religiões e que a } \\
\text { espiritualidade é benéfica, pouca } \\
\text { importância é dada à identidade } \\
\text { espiritual de seus pacientes, o que } \\
\text { pode dificultar uma abordagem } \\
\text { adequada ao } \\
\text { seu processo de morte. }\end{array}$ \\
\hline $\begin{array}{l}\text { No fio da navalha: a } \\
\text { dimensão } \\
\text { intersubjetiva do } \\
\text { cuidado aos bebês } \\
\text { com condições } \\
\text { crônicas complexas }\end{array}$ & 2019/Brasil & $\begin{array}{l}\text { SciELO/Physis: } \\
\text { Revista de } \\
\text { Saúde Coletiva }\end{array}$ & $\begin{array}{l}\text { Qualitativo /Qualitativa, } \\
\text { exploratória e } \\
\text { observacional ( } n=15 \\
\text { profissionais) }\end{array}$ & $\begin{array}{l}\text { Entrevista semiestruturada } \\
\text { sobre terminalidade em bebês } \\
\text { com condições crônicas } \\
\text { complexas. }\end{array}$ & $\begin{array}{l}\text { Reconheceu e valorizou os desafios } \\
\text { da prática de cuidado e as } \\
\text { repercussões psíquicas nos } \\
\text { profissionais de saúde. Valorização } \\
\text { da elaboração psíquica e da } \\
\text { produção de sentidos dos sujeitos. }\end{array}$ \\
\hline
\end{tabular}


Clasificación de la Association for

Children's Palliative

Care (ACT) en una

institución pediátrica

de referencia en el

Caribe colombiano

2018/Colômbia LILACS/Revista de Salud Pública

- Salud Pública

Quantitativo /Descritivo retrospectivo $(n=2718$ pacientes)
Revisão dos prontuários da população pediátrica de 0 a 18 anos atendida no Hospital

Infantil Napoleón Franco

Pareja, na cidade de

Cartagena, Colômbia, no

período de $1^{\circ}$ de janeiro de

2013 a 31 de dezembro de

2015.
Encontrada população suscetível aos cuidados paliativos pediátricos, para a qual foi necessário

estabelecer medidas de

complementação e integralidade do cuidado.

\section{(continua)}




\begin{tabular}{|c|c|c|c|c|c|}
\hline Título & Ano/País & \begin{tabular}{|l|} 
Base de \\
dados/Periódico
\end{tabular} & Estilo/Delineamento & Intervenção & Desfecho \\
\hline $\begin{array}{l}\text { Considerações } \\
\text { médicas, éticas e } \\
\text { jurídicas sobre } \\
\text { decisões de fim de } \\
\text { vida em pacientes } \\
\text { pediátricos }\end{array}$ & 2018/Brasil & $\begin{array}{l}\text { SciELO/Revista } \\
\text { Bioética }\end{array}$ & $\begin{array}{l}\text { Atualização /Artigo de } \\
\text { atualização }\end{array}$ & $\begin{array}{l}\text { Destaque para o processo de } \\
\text { tomada de decisões } \\
\text { referentes a cuidado de fim de } \\
\text { vida em pediatria. }\end{array}$ & $\begin{array}{l}\text { Fundamental o compartilhamento de } \\
\text { responsabilidades entre equipe de } \\
\text { saúde e pais, com a participação da } \\
\text { criança sempre que possível, } \\
\text { buscando o princípio do melhor } \\
\text { interesse e do direito de viver com } \\
\text { dignidade. }\end{array}$ \\
\hline $\begin{array}{l}\text { Empowering } \\
\text { Bereaved Parents } \\
\text { Through the } \\
\text { Development of a } \\
\text { Comprehensive } \\
\text { Bereavement } \\
\text { Program }\end{array}$ & $\begin{array}{l}\text { 2017/Estados } \\
\text { Unidos }\end{array}$ & $\begin{array}{l}\text { MEDLINE- } \\
\text { PubMed/J Pain } \\
\text { Symptom } \\
\text { Manage }\end{array}$ & $\begin{array}{l}\text { Descritivo /Descreve os } \\
\text { componentes de um } \\
\text { programa de luto e } \\
\text { apresenta os primeiros } \\
\text { resultados sobre os } \\
\text { efeitos de componentes } \\
\text { específicos do programa. }\end{array}$ & $\begin{array}{l}\text { Apresentação de relatório } \\
\text { sobre o programa }\end{array}$ & $\begin{array}{l}\text { Os serviços e intervenções } \\
\text { fornecidos por meio do programa de } \\
\text { luto são benéficos para as famílias } \\
\text { após a morte de seus filhos. Além } \\
\text { disso, tanto os profissionais de } \\
\text { saúde quanto os pais enlutados } \\
\text { relatam que a participação em } \\
\text { intervenções educacionais impacta } \\
\text { positivamente suas experiências } \\
\text { como médicos e pais, } \\
\text { respectivamente. }\end{array}$ \\
\hline $\begin{array}{l}\text { A espiritualidade na } \\
\text { prática pediátrica }\end{array}$ & 2016/Brasil & $\begin{array}{l}\text { SciELO/Revista } \\
\text { Bioética }\end{array}$ & $\begin{array}{l}\text { Atualização /Artigo de } \\
\text { atualização }\end{array}$ & $\begin{array}{l}\text { Destaque para a dimensão } \\
\text { espiritual, parte integrante do } \\
\text { cuidado ao paciente } \\
\text { pediátrico. }\end{array}$ & $\begin{array}{l}\text { A equipe de saúde precisa estar } \\
\text { apta a acolher o movimento de } \\
\text { transcendência e atenta às } \\
\text { necessidades espirituais desses } \\
\text { pacientes, bem como de seus } \\
\text { responsáveis }\end{array}$ \\
\hline
\end{tabular}




\begin{tabular}{|c|c|c|c|c|c|}
\hline $\begin{array}{l}\text { Personalized and yet } \\
\text { standardized: An } \\
\text { informed approach to } \\
\text { the integration of } \\
\text { bereavement care in } \\
\text { pediatric oncology } \\
\text { settings }\end{array}$ & $\begin{array}{l}\text { 2016/Estados } \\
\text { Unidos }\end{array}$ & $\begin{array}{l}\text { MEDLINE- } \\
\text { PubMed/Hispanic } \\
\text { Health Care } \\
\text { International }\end{array}$ & $\begin{array}{l}\text { Quantitativo /Estudo } \\
\text { quantitativo longitudinal ( } \mathrm{n} \\
=100 \text { participantes) }\end{array}$ & $\begin{array}{l}\text { Questionário sobre as } \\
\text { características do programa } \\
\text { pediátrico paliativo e de luto; } \\
\text { os desafios e barreiras para a } \\
\text { implementação dos padrões } \\
\text { de atendimento publicados. }\end{array}$ & $\begin{array}{l}\text { Poucos centros usam ferramentas } \\
\text { de avaliação do luto. Ausência de } \\
\text { avaliação de rotina das } \\
\text { necessidades de luto, duração } \\
\text { inconsistente dos cuidados ao luto e } \\
\text { muita variabilidade nos serviços, } \\
\text { sugerem que são necessários mais } \\
\text { estudos para promover o } \\
\text { atendimento ao luto padronizado e } \\
\text { orientado por políticas públicas. }\end{array}$ \\
\hline
\end{tabular}

(continua) 
(conclusão)

\begin{tabular}{|c|c|c|c|c|c|}
\hline Título & Ano/País & \begin{tabular}{|l|} 
Base de \\
dados/Periódico
\end{tabular} & Estilo/Delineamento & Intervenção & Desfecho \\
\hline $\begin{array}{l}\text { End-of-Life Care for } \\
\text { Hispanic Children: A } \\
\text { Study of California } \\
\text { Medicaid } \\
\text { Beneficiaries }\end{array}$ & $\begin{array}{l}\text { 2016/Estados } \\
\text { Unidos }\end{array}$ & $\begin{array}{l}\text { MEDLINE- } \\
\text { PubMed/Hispanic } \\
\text { Health Care } \\
\text { International }\end{array}$ & $\begin{array}{l}\text { Quantitativo /Estudo } \\
\text { quantitativo }(\mathrm{n}=370 \\
\text { participantes) }\end{array}$ & \begin{tabular}{l|} 
Aplicação de questionário \\
para avaliar a relação entre as \\
características da criança e da \\
família e a utilização de \\
cuidados de cuidados \\
paliativos ao final de vida.
\end{tabular} & $\begin{array}{l}\text { As práticas de enfermagem } \\
\text { destinadas a envolver as famílias } \\
\text { hispânicas em sua comunidade são } \\
\text { essenciais para a utilização dos } \\
\text { cuidados de final de vida para } \\
\text { crianças hispânicas }\end{array}$ \\
\hline $\begin{array}{l}\text { Cuidados paliativos e } \\
\text { espiritualidade: } \\
\text { revisão integrativa da } \\
\text { literatura }\end{array}$ & 2015/Brasil & $\begin{array}{l}\text { SciELO/Revista } \\
\text { Brasileira de } \\
\text { Enfermagem }\end{array}$ & $\begin{array}{l}\text { Revisão /Revisão } \\
\text { integrativa ( } \mathrm{n}=39 \\
\text { estudos) }\end{array}$ & $\begin{array}{l}\text { Busca nas bases de dados: } \\
\text { LILACS, MEDLINE/PubMed, } \\
\text { IBECS, SciELO. }\end{array}$ & $\begin{array}{l}\text { Relevância da dimensão espiritual } \\
\text { durante a assistência de pacientes } \\
\text { assistidos por meio de cuidados } \\
\text { paliativos e a necessidade do } \\
\text { desenvolvimento de novos estudos } \\
\text { para disseminar conhecimento sobre } \\
\text { o tema. }\end{array}$ \\
\hline
\end{tabular}


Palliative care in paediatric oncology:

perceptions, expertise

and practices from the

perspective of the

multidisciplinary team
SciELO/Revista

Gaúcha de

Enfermagem
Qualitativo /Qualitativa,

$\begin{array}{ll}\text { Ena, } & \text { Entrevista semiestruturada } \\ \text { sobre terminalidade em }\end{array}$

$=9$ profissionais) oncologia pediátrica.
A equipe sofre com a morte da criança, compartilha saberes para delinear as bases

do projeto terapêutico singular a ser

implementado e insere a família

nesse processo para que possa

assumir o protagonismo do cuidado

à criança. 


\section{DISCUSSÃO}

Após leituras sucessivas dos estudos selecionados para a presente revisão e o agrupamento de informações, foi possível construir quatro abordagens temáticas: Abordagem temática I - O significado do treinamento no campo dos cuidados paliativos; Abordagem temática II - Cuidados paliativos e luto; Abordagem temática III - A espiritualidade e o paciente sob cuidados paliativos e Abordagem temática IV - Cuidados paliativos e aspectos éticos.

\section{Abordagem temática I - 0 significado do treinamento no campo dos cuidados paliativos}

Estima-se que 40 milhões de pessoas necessitam de cuidados paliativos, contudo, apenas $14 \%$ os recebem, o que pode ser justificado pela falta de conhecimento, treinamento e conscientização por parte dos profissionais da saúde. ${ }^{9}$

Sabe-se que o treinamento das equipes de atenção à saúde precisa ter qualidade ótima e, ainda assim, várias ocorrências podem passar despercebidas e serem negligenciadas. ${ }^{10}$ Normalmente as equipes de saúde atendem a demandas diversas e não se sentem preparadas para a complexidade dos cuidados paliativos. ${ }^{10,11}$

Treinamento da equipe e educação permanente são os pilares para a qualidade assistencial, pois todos precisam ter muita segurança nos conhecimentos inerentes à prática proposta. ${ }^{12,13}$

É notável a falta capacitação tanto da família dos pacientes pediátricos em cuidados paliativos, quanto da própria e a equipe de atenção à saúde sobre o processo de paliação faz com que o cuidado seja dificultado. Sendo assim, fazse necessário que as equipes multiprofissionais, bem como todos os acompanhantes e familiares envolvidos no cuidado às crianças e adolescentes, recebam treinamento sobre do assunto. ${ }^{14}$ Ademais, é de grande relevância a instalação de protocolos sobre cuidados paliativos em instituições de saúde. ${ }^{14}$, 15

\section{Abordagem temática II - Cuidados paliativos e luto}

Sabe-se que o cuidado paliativo pediátrico é caracterizado por uma assistência ativa e integral, perpassando pelas ações junto ao corpo propriamente dito, a mente e ao espírito da criança, além de abranger aspectos da prestação de apoio à família, inclusive no período do luto. ${ }^{16}$

Percebe-se que sentimentos paradoxais, como justiça/injustiça, querer que viva/querer que morra, sentir/não sentir, chorar/não chorar, dizer algo/calar, são um reflexo de uma sociedade que nega o sofrimento através da supressão do luto, e que transforma as instituições de cuidado em saúde em esconderijos da morte e do sofrimento. ${ }^{17,} 18$ 
A prevalência de sistemas hierarquização e normativos, presentes principalmente em instituições hospitalares, dificultam a comunicação e o diálogo entre os colaboradores da equipe multiprofissional envolvidos no processo da morte e do morrer. ${ }^{19}$

Associado a isso, tem-se uma rotina acelerada e múltiplas demandas de cuidado o que dificulta a criação de momentos para a troca entre os profissionais, os quais se sentem imersos em um fazer sistemático e cansativo. ${ }^{20}$

Contudo, acredita-se que, para esses colaboradores que lidam sistematicamente com a interface entre a dor e a morte, é fundamental maior elaboração desses sentimentos para que o luto deles também possa ser trabalhado de maneira sadia e respeitosa. ${ }^{20}$ Nesse sentido, é necessário que toda a equipe multiprofissional consiga trabalhar os sentimentos pessoais para que seja possível lidar com as questões do luto na pediatria das crianças e adolescentes em cuidados paliativos. ${ }^{20,21}$

\section{Abordagem temática III - A espiritualidade e o paciente sob cuidados paliativos}

Outro desafio importante é a falta de destreza para abordar e compreender aspectos que envolvem a espiritualidade. ${ }^{22}$ As questões individuais e coletivas sobre finitude, religião e espiritualidade são o ponto de partida para auxiliar melhor os pacientes em cuidados paliativos, principalmente em pediatria, pois os dilemas relacionados à terminalidade da vida em pediatria ocupam relevante espaço de discussão. ${ }^{8,22}$

Nesse contexto, a atenção à espiritualidade se torna cada vez mais necessária na prática de assistência à saúde, independente da faixa etária, pois vem sendo reconhecida como fonte de bem-estar e de qualidade de vida ao se aproximar a morte. ${ }^{22,}{ }^{23}$ Para tanto, é fundamental que a equipe de saúde esteja apta a acolher esse movimento de transcendência. ${ }^{22} \mathrm{~A}$ equipe assistencial deve ser treinada para aceitar os diferentes valores religiosos e espirituais, não impondo conceitos próprios, mas respeitando e incentivando a participação do paciente em sua prática. ${ }^{11,21}$

Em pediatria, a definição de terminalidade, por representar situação mais trágica, é mais difícil do que no adulto, de modo que a determinação da irreversibilidade na criança e/ou adolescente é um processo mais duro, que demanda mais tempo. ${ }^{22}$ Esse fato ocorre especialmente nas nações da América Latina, pois nestes países latinos a medicina se caracteriza fortemente por deter um componente paternalista, além de um conceito tradicional de manutenção da vida a qualquer preço, tanto do ponto de vista da sociedade civil quanto da prática médica. ${ }^{22}$

\section{Abordagem temática IV - Cuidados paliativos e aspectos éticos}


A evolução dos aspectos médicos e assistências em saúde, com aprimoramento e desenvolvimento tecnológico, ao mesmo tempo que permite maior acurácias para os diagnóstico e melhoria para opções de tratamentos precoces de um espectro cada vez maior de doenças, altera a história natural dessas enfermidades, o que torna os prognósticos tarefa ainda mais complexa. ${ }^{24}$

A aplicação do conhecimento de um arsenal de possibilidades para a manutenção da vida, ainda não está em acordo com as consequências de ações ao empregá-lo ${ }^{24,25}$, além de também não ter estudos suficientes para entender a representação para as famílias, em médio e longo prazos, da opção pelo uso de equipamentos de suporte artificial de vida capazes de substituir de forma permanente as funções vitais. ${ }^{24}$

O aumento da disponibilidade de recursos tecnológicos para a manutenção da vida de crianças e/ou adolescentes com doenças incuráveis tem potencializado conflitos entre os próprios integrantes da equipe multiprofissional de saúde e destes com as famílias ${ }^{24,26}$, de modo a promover, por vezes, o processo de judicialização da saúde, geralmente com consequências desastrosas para todas as partes envolvidas, uma vez que as relações entre médico-pacientefamília são pautadas por nuances pouco acessíveis aos julgadores. ${ }^{24}$

Percebe-se que a abordagem humanista da relação equipe de saúde-pacientefamília, sobretudo em situações relacionadas a cuidados em pediatria de pacientes com patologias progressivas e incuráveis, deve ser uma constante. ${ }^{27}$

Todavia, torna-se fundamental que, diante do aprimoramento das técnicas de comunicação e da capacidade empática de cuidar, a equipe de saúde seja capaz de resolver mais conflitos, buscando consenso com os acompanhantes e familiares na tentativa de se evitar intervenção jurídica. ${ }^{28}$

\section{CONSIDERAÇÕES FINAIS}

Os artigos examinados neste estudo sobre cuidados paliativos e pediatria mostraram existem possibilidades de recuperar a qualidade de vida dos pacientes por meio da melhoria do bem-estar, mediante o alívio da dor e de outros sintomas.

Apesar de ser ainda pouco difundida no Brasil, a abordagem de cuidados paliativos vem ganhando cada vez mais espaço no país, principalmente em grandes centros urbanos. Contudo, é necessário o desenvolvimento de novas pesquisas, principalmente no âmbito nacional, para se respaldar 0 atendimento da equipe multiprofissional que assistem pacientes pediátricos em cuidados paliativos.

Também é iminente a realização de estudos que investiguem os instrumentos de avaliação direcionados para pacientes sob cuidados paliativos e sua adaptação transcultural, visto que, em nosso estudo, alguns instrumentos podem não ter sido abordados. 


\section{REFERÊNCIAS}

1. Boff L. Saber cuidar: ética do humano: compaixão pela terra. 9. ed. Petrópolis: Vozes; 2003.

2. World Health Organization. National cancer control programs: policies and managerial guidelines. 2. ed. Geneva: WHO; 2002.

3. World Health Organization. Palliative care [Internet]. Genebra: WHO; 19 Fev 2020. Disponível em: https://www.who.int/en/news-room/ factsheets/detail/palliative-care

4. Vasconcelos GB, Pereira PM. Cuidados paliativos em atenção domiciliar: uma revisão bibliográfica. Rev. Adm. Saúde, 2018; 18(70). http://dx.doi.org/10.23973/ras.70.85

5. Merino MTGB. Palliative care: taking the long view. Front Pharmacol. 2018; 9:1140. DOI: https://doi.org/10.3389/fphar.2018.01140.

6. Costa de Oliveira L. Cuidados Paliativos: Por que Precisamos Falar sobre isso? Rev. Brasileira De Cancerologia [Internet]. 16 Dez 2019; 65(4):e-4558. Disponível em: https://rbc.inca.gov.br/revista/index.php/revista/article/view/558

7. Branco MG, Santos HS. Família e Cuidados Paliativos em Pediatria: Desafios à Garantia do Cuidado. Rev. Brasileira De Cancerologia [Internet]. 30 Set 2016; 62(3):259-62. Disponível em: https://rbc.inca.gov.br/revista/index.php/revista/article/view/339

8. Evangelista CB, Lopes MEL, Costa SFG, Batista PSS, Batista JBV, Oliveira AMM. Cuidados paliativos e espiritualidade: revisão integrativa da literatura. Rev. Bras. Enferm. [Internet]. Jun 2016; 69(3):591-601. Disponível em: http://www.scielo.br/scielo.php?script=sci arttext\&pid=S0034$\underline{71672016000300591 \& \operatorname{lng}=\text { en }}$

9. OMS. Manual de cuidados paliativos. 2014. Disponível em: http://www.who.int. Acesso em: 26 ago. 2020.

10. Azevedo CDS, Pfeil NV. No fio da navalha: a dimensão intersubjetiva do cuidado aos bebês com condições crônicas complexas. Physis [Internet]. 2019; 29(4):e290406. Disponível em: http://www.scielo.br/scielo.php?script=sci arttext\&pid=S010373312019000400604\&lng $=\mathrm{pt}$

11.Salvador Coloma C., Salvador Coloma V., Segura Huerta A., Andrés Moreno M., Fernández Navarro J. M., Niño Gómez O. M. et al. Actualidad de los cuidados paliativos: revisión de una situación poco estandarizada. Rev Pediatr Aten Primaria [Internet]. 2015 Sep; 17(67):e215-e222. 
Disponible en: http://scielo.isciii.es/scielo.php?script=sci arttext\&pid=S1139$\underline{76322015000400017 \& \operatorname{lng}=\mathrm{es}}$

12. Carvalho RT, Parsons HA. Manual de Cuidados Paliativos ANCO. 2. ed. São Paulo: ANCP; 2012.

13. Flórez SP, Tovar MB, León MX, Villegas K, Villamizar D del P, Granados CE. Caracterización del conocimiento en cuidado paliativo pediátrico y percepción de barreras por parte de los pediatras y residentes de pediatría. MEDIPAL. 2015; 22:127-35.

14. Lindley LC, Trujillo LV. End-of-Life Care for Hispanic Children: A Study of California Medicaid Beneficiaries. Hisp Health Care Int. 2016 Dec; 14(4):164-169. DOI: http://dx.doi.org/10.1177/1540415316670900

15. Tirado-Perez Irina S., Zarate-Vergara Andrea C. Clasificación de la Association for Children's Palliative Care (ACT) en una institución pediátrica de referencia en el Caribe colombiano. Rev. salud pública [Internet]. 2018 June; 20(3): 378-383. Available from: http://www.scielo.org.co/scielo.php?script=sci arttext\&pid=S012400642018000300378\&lng=en

16. Organização Mundial de Saúde $(\mathrm{CH})$ [Internet]. Cuidados paliativos em pediatria. Disponível em: http://www.who.int/cancer/palliative/es/index.htm

17. Wiener L, Rosenberg AR, Lichtenthal WG, Tager J, Weaver MS. Personalized and yet standardized: An informed approach to the integration of bereavement care in pediatric oncology settings. Palliat Support Care. 2018 Dec; 16(6):706-711. DOI: http://dx.doi.org/10.1017/S1478951517001249

18. Silva AF, Issi HB, Motta MGC, Botene DZA. Palliative care in pediatric oncology: perceptions, expertise and practices from the perspective of the multidisciplinary team. Rev. Gaúcha Enferm. [Internet]. 2015 Jun; 36(2): 5662. Disponível em: http://www.scielo.br/scielo.php?script=sci arttext\&pid=S1983$14472015000200056 \& \operatorname{lng}=\mathrm{pt}$

19. Borges MS, Mendes N. Representações de profissionais de saúde sobre a morte e o morrer. Rev Bras Enferm.2012; 65(2):324-31.

20. Carreño-Moreno Sonia, Chaparro-Díaz Lorena, López-Rangel Rocío. Encontrar sentido para continuar viviendo el reto al perder un hijo por cáncer infantil: revisión integrativa. pers.bioét. [Internet]. 2017 Jan; 21(1): 46-61. Available from: http://www.scielo.org.co/scielo.php?script=sci arttext\&pid=S0123$\underline{31222017000100046 \& \operatorname{lng}=e n}$

21. Snaman JM, Kaye EC, Levine DR, Cochran B, Wilcox R, Sparrow CK, Noyes N, Clark L, Avery W, Baker JN. Empowering Bereaved Parents Through the Development of a Comprehensive Bereavement Program. J 
Pain Symptom Manage. 2017 Apr; 53(4):767-775. DOI: http://dx.doi.org/10.1016/j.jpainsymman.2016.10.359

22. Garanito MP, Cury MRG. A espiritualidade na prática pediátrica. Rev. Bioét. [Internet]. 2016 Apr; 24(1):49-53. Available from:

http://www.scielo.br/scielo.php?script=sci arttext\&pid=S198380422016000100049\&lng $=$ en

23. Naufel LZ, Sarno MTCD, Alves MAJ. Physicians' knowledge about patients' religious beliefs in pediatric care. Rev. paul. pediatr. [Internet]. 2019 Dez; 37 4) 479-485. Disponível em: http://www.scielo.br/scielo.php?script=sci arttext\&pid=S010305822019000400479\&lng $=\mathrm{pt}$

24. Dadalto L, Affonseca CA. Considerações médicas, éticas e jurídicas sobre decisões de fim de vida em pacientes pediátricos. Rev. Bioét. [Internet]. 2018 Jan; 26(1):12-21. Disponível em:

http://www.scielo.br/scielo.php?script=sci arttext\&pid=S198380422018000100012\&lng=pt

25. Bernadá M, Notejane M, Martínez R, Campos C. Opinión de los profesionales de la salud sobre un documento de registro de directivas de adecuación de esfuerzo terapéutico en pediatría. Rev. Méd. Urug. 2020; 36(2): 36-58. Disponible en:

http://www.scielo.edu.uy/scielo.php?script=sci arttext\&pid=S168803902020000200036\&lng=es

26. D'Acosta L, Hermida N, Urrutia M, Notejane M, Bernadá M, Dall'Orso P. Consultas de niños pasibles de cuidados paliativos al Departamento de Emergencia Pediátrica del Centro Hospitalario Pereira Rossell 2014- 2015. Arch Pediatr Urug. 2016; 87(4):332-41.

27. Konder CNP, Teixeira ACB. Crianças e adolescentes na condição de pacientes médicos: desafios da ponderação entre autonomia e vulnerabilidade. Pensar. 2016; 21(1):70-93.

28. Bradley C, Brasel K, Schwarze M. Physician attitudes regarding advance directives for high-risk surgical patients: a qualitative analysis. Surgery. 2010; 148(2):209-16.

Recebido: 12 de outubro de 2020. Aceito: 24 de dezembro de 2020

Correspondência: Luciana Alves Silveira Monteiro. E-mail:

luciana.silveira.monteiro@gmail.com 
Conflito de Interesses: os autores declararam não haver conflito de interesses.

(C) This is an Open Access article distributed under the terms of the Creative Commons Attribution License, which permits unrestricted use, distribution, and reproduction in any medium, provided the original work is properly cited 